\title{
UCRL-BOOK-206080
}

LAW RENCE LIVERMORE N A T IO N A L LABORATORY

Bridging the Gap between

Quantum Mechanics and

Large-Scale Atomistic Simulation

J. A. Moriarty

August 16, 2004

Handbook of Materials Modeling 
This document was prepared as an account of work sponsored by an agency of the United States Government. Neither the United States Government nor the University of California nor any of their employees, makes any warranty, express or implied, or assumes any legal liability or responsibility for the accuracy, completeness, or usefulness of any information, apparatus, product, or process disclosed, or represents that its use would not infringe privately owned rights. Reference herein to any specific commercial product, process, or service by trade name, trademark, manufacturer, or otherwise, does not necessarily constitute or imply its endorsement, recommendation, or favoring by the United States Government or the University of California. The views and opinions of authors expressed herein do not necessarily state or reflect those of the United States Government or the University of California, and shall not be used for advertising or product endorsement purposes. 


\section{Bridging the Gap between Quantum Mechanics and Large-Scale Atomistic Simulation}

The prospect of modeling across disparate length and time scales to achieve a predictive multiscale description of real materials properties has attracted widespread research interest in the last decade. (See, for example, Moriarty et al., 2002a and the references therein.) To be sure, the challenges in such multiscale modeling are many, and in demanding cases, such as mechanical properties or dynamic phase transitions, multiple bridges extending from the atomic level all the way to the continuum level must be built. Although often overlooked in this process, one of the most fundamental and important problems in multiscale modeling is that of bridging the gap between first-principles quantum mechanics, from which true predictive power for real materials emanates, and the large-scale atomistic simulation of thousands or millions of atoms, which is usually essential to describe the complex atomic processes that link to higher length and time scales. For example, to model single-crystal plasticity at micron length scales via dislocation-dynamics simulations that evolve the detailed dislocation microstructure requires accurate large-scale atomistic information on the mobility and interaction of individual dislocations. Similarly, modeling the kinetics of structural phase transitions requires linking accurate large-scale atomistic information on nucleation processes with higher length and time scale growth processes.

\section{Electronic-Atomic Gap}

As indicated in Figure 1, there currently exists a wide spectrum of atomic-scale simulation methods in condensed-matter and materials physics, extending from essentially exact quantum-mechanical techniques to classical descriptions with totally empirical force laws. All of these methods fall into one of two distinct categories, which are separated by a material-dependent gap. On one side of this gap are electronic methods based on direct quantum-mechanical treatments. These include quantum simulations that attempt to treat electron and ion motion on an equal footing, solving quantum-mechanical equations on the fly for both the electronic states of the system and the forces on the individual ions. In principle, such methods can provide a highly accurate description of the system and are chemically very robust, but they come at the price of being severely limited in the size and

duration of the simulation. Typically, even efficient mean-field methods such as quantum 
molecular dynamics (QMD) (Car and Parrinello, 1985; Payne et al., 1992) can at best treat a hundred or so atoms for a few picoseconds of time.

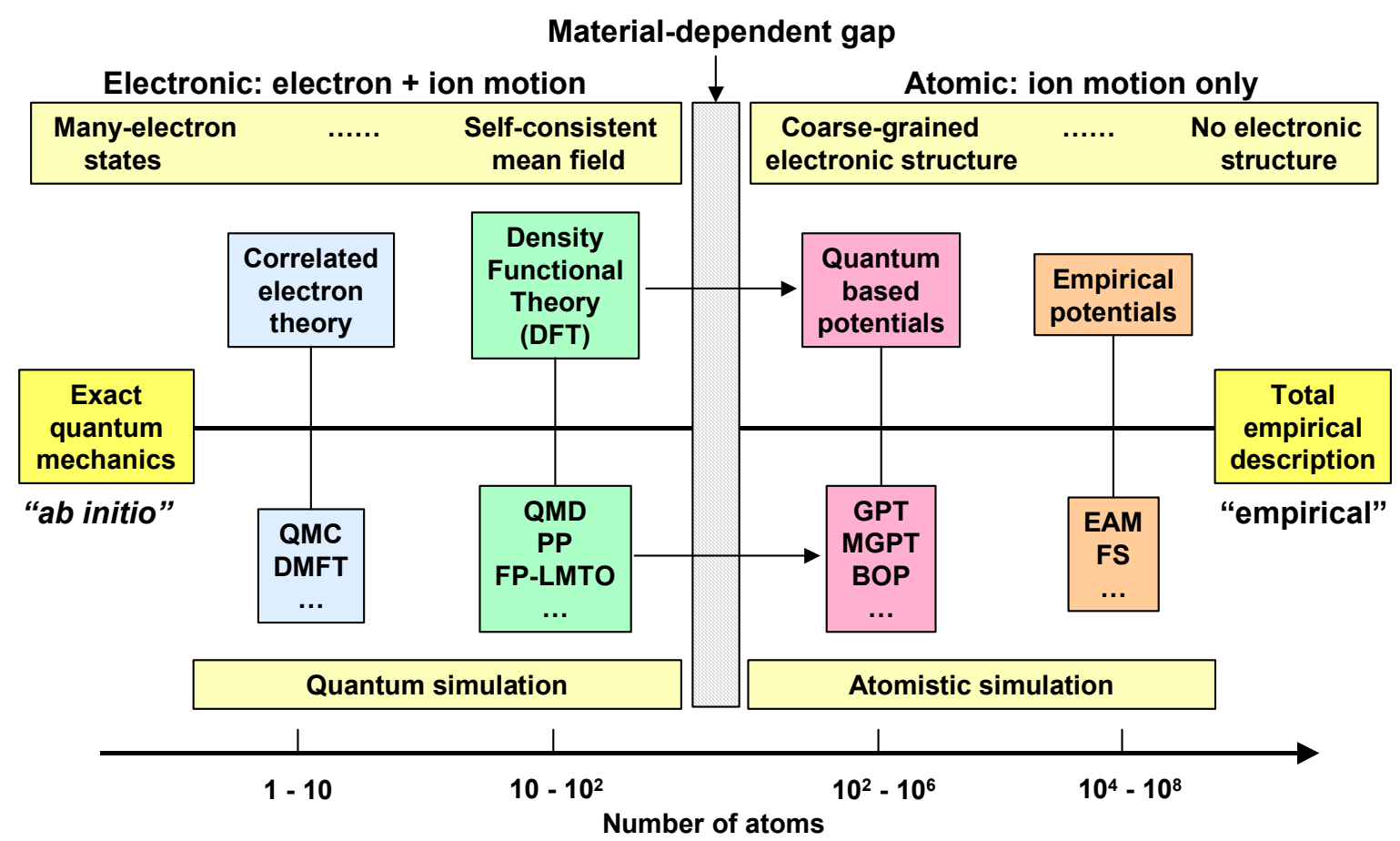

Figure 1. Representative sample of the wide spectrum of electronic and atomistic simulation approaches used in condensed-matter and materials physics and the materialdependent gap separating them.

On the other side of the gap are methods used in atomistic simulations that treat only the ion motion, solving classical Newtonian equations of motion with the forces derived from explicit interatomic potentials, which may or may not be encoded with detailed quantum information about the electronic structure. For the simplest short-range empirical potentials, tens or hundreds of millions of atoms can be so simulated with molecular dynamics (MD) for time durations extending to tens or hundreds of nanoseconds. But this computational robustness often comes at the price of losing any connection to the underlying electronic structure of the material. For studying generic phenomena in simple systems this may not be a major drawback, but more generally, for the predictive multiscale modeling of real complex materials that we envision here, the retention of adequate quantum information is essential. 
In practice, both quantum and atomistic simulations may be approached at many different levels of approximation. Many-body quantum-mechanical methods attempt to treat the full many-electron states of the system and provide a general means of addressing the fundamental issue of electron correlation. The valence electrons in the vast majority of systems of practical interest, however, including most metals and semiconductors, effectively exhibit only weak electron correlation. For such systems accurate total energies and forces can be achieved through self-consistent, mean-field electronicstructure methods based on modern density functional theory (DFT) (Hohenberg and Kohn, 1964; Kohn and Sham, 1965). Indeed, today DFT-based electronic-structure and quantum simulation methods are usually described as "ab initio," even though significant approximations from exact quantum mechanics are involved. Nonetheless, for weakly correlated systems first-principles DFT methods are quantitatively predictive and rely on only the barest input information: the atomic numbers and masses of the material constituents. Within this category of computational method are all-electron, full-potential (e.g., FP-LMTO) techniques as well as pseudopotential (PP) techniques, which treat only valence electrons and are normally essential in QMD simulations. In addition to such direct computational approaches, simplified representations of DFT are also possible via orbital-basis-state approaches using plane waves, localized atomic orbitals or a hybrid combination of the two. Such simplification provides a useful starting point to "coursegrain" the electronic structure and actually bridge the electronic-atomic gap.

The interatomic potentials used in atomistic simulations have likewise been developed to many different levels of sophistication and quantum compatibility. One basic consequence of the quantum-mechanical nature of electronic bonding in both metals and semiconductors is that the total energy of the system, $E_{t o t}\left(R_{1} \ldots R_{N}\right)$, is inherently a manybody functional of its atomic coordinates $R_{i}$ and must contain terms beyond radial-force pair interactions. This requirement is directly manifest in a number of measurable properties including the elastic moduli, where in metals it is well known that the Cauchy relations implied by pure pair potentials are not satisfied in general (e.g., $C_{12} \neq C_{44}$ in cubic metals) and in semiconductors non-radial forces are needed even to stabilize the basic diamond structure. Most modern empirical potentials satisfy this requirement by adding a more general functional to a pair-potential contribution in $E_{t o t}$. In some cases, this additional contribution is inspired by specific quantum-mechanical considerations, but typically arbitrary short-ranged functional forms are still maintained in both pair and non-pair terms. In addition to such empirical potentials, however, there are also more 
rigorous quantum-based interatomic potentials (QBIPs) - potentials that are actually derived in whole or in part from the underlying quantum mechanics by suitably course graining of the electronic structure. The gap between electronic and atomistic methods and between QMD and MD/QBIP simulations is then directly related to the additional approximations entailed in such course graining. The size of the gap and our ability to bridge it is dependent both on the complexity of the material in question and the complexity of its environment. For some materials and/or some environments this gap is actually relatively small and can be readily bridged, such as is the case for bulk simple metals. For other materials and/or environments, however, the gap is larger and bridging it is still a forefront challenge of current research. This is the case, for example, with directionally-bonded transition metals and semiconductors as well as for chemically reactive surfaces. Nonetheless, significant progress has been made in the last decade or so in many of these latter areas, inspired in part by the demands of multiscale modeling.

\section{Quantum-Based Interatomic Potentials}

In the case of simple $s p$-bonded metals (e.g., $\mathrm{Na}, \mathrm{Mg}, \mathrm{Al}$ ), a rigorous formulation of QBIPs in the bulk material has been available since the 1960s in terms of pseudopotential perturbation theory (Harrison, 1966). In this approach, it is recognized that the electronic structure of such materials is nearly free electron in character and that the electron-ion interaction can be represented by a weak nonlocal pseudopotential. Using an orbital basis of plane waves, one can develop $E_{t o t}$ to second-order in the pseudopotential and express the result explicitly in the real-space form

$$
E_{t o t}\left(R_{1} \ldots R_{N}\right)=N E_{v o l}(\Omega)+\frac{1}{2} \sum_{i, j}^{\prime} v_{2}\left(R_{i j} ; \Omega\right),
$$

where $\Omega$ is the atomic volume of the metal, $E_{v o l}$ is a collective volume term that satisfies the many-body requirement for $E_{\text {tot }}$, and $v_{2}$ is a volume-dependent, but structureindependent and transferable, radial-force pair potential. The volume dependence of $v_{2}$ is a consequence of the self-consistent electron screening, which also gives rise to longrange Friedel oscillations in the potential tail. By the 1970s and 80s, first-principles DFT-based implementations of this approach had already become well developed (Dagens, Rasolt and Taylor, 1975; McMahan and Moriarty, 1982; Hafner, 1987). This method is particularly effective in dealing with bulk structural properties, including phonons and elasticity, solid phase transitions, liquid structure and dynamics, and 
melting. This approach has also been readily and successfully extended to compounds and alloys as well as to high pressures, where atomic volume is a very compatible environmental variable.

In the $1980 \mathrm{~s}$, interest in simulating materials properties beyond the bulk environment and particularly at surfaces led to the development of alternative "glue" models for simple metals (Nieminen, Puska and Manninen, 1990). These include the radial-force potential models obtained from the embedded-atom method (EAM) (Daw, Foiles and Baskes, 1993) and from effective medium theory (EMT) (Jacobsen, Norskov and Puska, 1987). The total-energy functional in the EAM or EMT is inspired by the DFT notion that the total energy of a system is a functional of its electron density and is assumed to take the form of an attractive embedding contribution balanced by a repulsive pair-potential contribution:

$E_{t o t}\left(R_{1} \ldots R_{N}\right)=\sum_{i} F\left(\bar{n}_{i}\right)+\frac{1}{2} \sum_{i, j}^{\prime} v_{2}\left(R_{i j}\right)$

where $F$ is a nonlinear function of the average electron density $\bar{n}_{i}$ on the site $i$. The embedding contribution correctly accounts for the increased bond strength at the surface relative to the bulk, although Eq. (2) itself is an ansatz and can't be directly derived from quantum mechanics. In the EMT, the ingredients of this equation are evaluated from first-principles DFT considerations within a well-defined prescription starting from the embedding of an atom in a free-electron gas. In the EAM, on the other hand, these ingredients are all treated empirically with convenient parameterized analytic forms chosen for $F, \bar{n}_{i}$ and $v_{2}$ to maximize flexibility and achieve high computational speed. Alternatively, $F, \bar{n}_{i}$ and $v_{2}$ have also been spline-fit to larger databases that also include DFT energies and forces. Regardless of how they are parameterized, the EAM and EMT models are most appropriate for simple $s p$-bonded metals and series-end transition metals (e.g., $\mathrm{Cu}$ ), where a description of the bonding in terms of radial forces is reasonable, and to such systems these models been extensively applied in the past twenty years.

A general approach to QBIPs that does allow one to go beyond radial-force interactions in a rigorous way makes use of a local-orbital, tight-binding (TB) representation of the electronic structure (Pettifor, 1995). In principle, if an accurate TB representation can be found, one can then develop a QBIP model based on an expansion of the total energy in 
terms of moments of the local electronic density of states, $\mu_{2}, \mu_{3}, \mu_{4} \ldots$. In practice, the notion of casting potentials in terms of moments has been used both to develop empirical models as well as in full quantum-mechanical derivations. Such approaches have been mainly directed at the $d$ states in central transition metals (e.g., Mo) and at the $s$ and $p$ states in covalently bonded semiconductors (e.g., Si). The simplest empirical scheme in this category is the second-moment, radial-force Finnis-Sinclair (FS) model (1984). This model is formally similar to the "glue" models discussed above with an assumed embedding function in the form $F\left(\mu_{2}\right) \propto\left(\mu_{2}\right)^{1 / 2}$, where the second moment $\mu_{2}$ is treated empirically as a short-ranged radial function about each atomic site. The FS model has been mostly applied to central bcc transition metals, although at this level of treatment there are in fact no angular-force terms to accommodate the $d$-state directional bonding. In this regard, empirical fourth-moment schemes for transition metals have also been developed that do implicitly include angular- as well as radial-force contributions. The most complete and fundamental TB approach to QBIPs, however, is the bond-orderpotential (BOP) model of Pettifor (1995), which is based on an explicit expansion of the total energy within TB theory and has been considerably developed and applied over the last ten years (Mrovec et al., 2004). In all the TB schemes, an empirical repulsive pairpotential contribution is included in the total energy, as in Eq. (2), and parameterization of the local-orbital matrix elements defining the moments is required. In the BOP model, the embedding energy of Eq. (2) is directly replaced by the full TB bond energy derived from quantum mechanics for the dominant bonding electrons, e.g., the $d$ electrons in transition metals. In such a case an additional empirical environmental energy correction term to the pair potential is also added to $E_{\text {tot }}$ to account for the fact that local $s$ and $p$ orbitals in a TB representation of the $s p$ electrons are effectively environmentally dependent.

Another general approach to QBIPs in metals involves combining a plane-wave-based pseudopotential treatment for $s$ and $p$ electrons with a local-orbital-based tight-binding treatment for $d$ electrons, allowing application to both simple and transition metals. The primary example of this approach is first-principles generalized pseudopotential theory (GPT), which has been rigorously developed from DFT quantum mechanics (Moriarty, 1988). In the GPT applied to transition metals, a mixed basis of plane waves and localized $d$-state orbitals is used to self-consistently expand the electron density and total energy of the system in terms of weak $s p$ pseudopotential, $d$ - $d$ tight-binding, and $s p-d$ hybridization matrix elements, which in turn are all directly calculable from first principles. The GPT total-energy expansion has been carried out to the level of four-ion 
interactions and formally generalizes Eq. (1). In a bulk elemental transition metal, one obtains the explicit real-space form

$$
E_{t o t}\left(R_{1} \ldots R_{N}\right)=N E_{v o l}(\Omega)+\frac{1}{2} \sum_{i, j}^{\prime} v_{2}(i j ; \Omega)+\frac{1}{6} \sum_{i, j, k}^{\prime} v_{3}(i j k ; \Omega)+\frac{1}{24} \sum_{i, j, k, l}^{\prime} v_{4}(i j k l ; \Omega)
$$

The leading volume term in this expansion, $E_{v o l}$, as well as the two-, three-, and four-ion interatomic potentials, $v_{2}, v_{3}$, and $v_{4}$, are as in Eq. (1) volume dependent, but structure independent quantities and thus transferable to all bulk ion configurations, either ordered or disordered. This includes all structural phases as well as the deformed solid and the imperfect bulk solid with either point or extended defects present. The angular-force multi-ion potentials $v_{3}$ and $v_{4}$ in Eq. (3) reflect contributions from partially-filled $d$ bands and are generally important for central transition metals. In the full ab initio GPT, however, these potentials are long-ranged, nonanalytic and multidimensional functions, so that $v_{3}$ and $v_{4}$ cannot be readily tabulated for application purposes. This has led to the development of a simplified and complementary model GPT or MGPT applicable to central transition metals (Moriarty et al., 2002b). Within the MGPT, the multi-ion potentials $v_{3}$ and $v_{4}$ are systematically approximated by introduing canonical $d$ bands and other simplifications to achieve short-ranged, analytic forms, which can then be applied to large-scale atomistic simulations. To compensate for the approximations introduced into the MGPT, a limited amount of parameterization is allowed in which the coefficients of the modeled potential terms are constrained by either DFT or experimental data.

In practice, the $a b$ inito GPT and the MGPT potentials have complementary ranges of application. The ab initio GPT is most effective in situations where the total-energy expansion (3) can be truncated at the pair-potential level, as in Eq. (1), since tabulation and interpolation of a nonanalytic pair potential $v_{2}(r, \Omega)$ represents no computational barrier for atomistic simulations. Thus ab initio GPT applications include simple metals and series-end transition metals as well as appropriate binary alloys, including the transition-metal aluminides (Moriarty and Widom, 1997). The primary application range for the MGPT, on the other hand, is the bcc transition metals (e.g., Ta, Mo). Both GPT and MGPT potentials have been implemented in atomistic simulations and applied to a wide range of bulk structural, thermodynamic, defect and mechanical properties at both ambient and extreme conditions of temperature and pressure (Moriarty et al., 2002b). Extension of the bulk GPT and MGPT potentials to highly nonbulk situations, such as 
surfaces, voids and clusters, is also possible through appropriate environmental modulation (Moriarty and Phillips, 1991). This refinement has only been studied in detail, however, in the cases of free surfaces, where environmental corrections have been shown to be very important ( 50-70\%), and for vacancies, where such corrections have been confirmed to be negligible $(\sim 1-2 \%)$.

\section{Outlook}

There are still many remaining challenges in bridging the gap between quantum and atomistic simulations. One inherent advantage that QBIPs have over empirical potentials in this quest is that they are systematically improvable in a manner consistent with quantum mechanics. In spite of the significant progress made over the past forty years, the collective amount of time and energy spent to date on developing QBIPs has actually been very small compared to that spent on developing advanced electronic-structure methods and quantum simulations themselves. As a result, this is still a young research field that has very much room to grow. Below we discuss three general areas where there would seem to be great opportunities for major progress over the next decade:

\section{(i) improved accuracy and computational speed}

In first-principles QBIPs such as GPT, the main additional approximation beyond DFT is the truncation of the total-energy expansion at finite order. For both simple and transition metals, it is now computationally feasible to extend this expansion to higher order as needed. For transition metals in particular, it should be possible to expand Eq. (3), both in the GPT and MGPT representations, to include five- and six-ion $d$-state interactions. This would improve the description of certain structural properties, such as the hcp-fcc energy difference and corresponding stacking faults, and would enable accurate applications both to the left and right of the central bcc metals. Moreover, in the context of semi-empirical QBIPs such as MGPT or BOP, it should be possible to eliminate or improve many of the secondary approximations that are currently used. In the MGPT, for example, it has recently been possible to formulate a more general matrix version of the theory that allows one to go beyond simple canonical $d$ bands and hence achieve a more accurate representation of the electronic structure. Also, in both MGPT and BOP current applications to transition metals, explicit $s p$ - $d$ hybridization contributions have been dropped for convenience, but these should be included in the future, especially for non-central transition metals. 
The balancing consideration to increased accuracy is, of course, computational speed. One enormously appealing aspect of empirical EAM potentials is their extreme speed, which can be up to six orders of magnitude faster than first-principles DFT electronicstructure or QMD methods and up to two orders of magnitude faster than QBIPs. While angular-force QBIPs will be inherently slower than radial-force EAM potentials, a reasonable goal would be to come within one order of magnitude of their speed, at least at some basic level of operation. In the case of MGPT, recent algorithm improvements have increased computational speed dramatically by up to a factor of six and put us at or close to that goal. Adding higher-order interactions and/or $s p$ - $d$ hybridization will, of course, work to reverse that gain, but inevitably one must think in terms of having QBIPs at various levels of approximation and match the level and speed with the intended application.

\section{(ii) treatment of complex systems and complex environments}

The general application area of intermetallic compounds and alloys would seem to a potentially ideal one for QBIPs since in general they are much better positioned to handle chemical and structural complexity than empirical potentials. In this regard, BOP and GPT treatments of transition-metal intermetallics have already had significant success and these applications are expected to continue and grow in the future. Future MGPT and hydrid MGPT/GPT treatments also look very promising for transition-metal rich systems. Similarly, another fruitful application area is expected to be high-pressure physics. Here volume-dependent GPT and MGPT treatments to date have proven to be successful, both in terms of predicting new high-pressure phases and in describing how materials properties scale with pressure for an existing phase. More generally, QBIPs seem to be well suited to deal with changes in structural stability under pressure and the prediction of the thermodynamics and mechanical properties of new encountered phases.

The application of QBIPs to non-bulk environments, such as free surfaces, voids and clusters, would also seem to be a potentially important area, especially for doing largescale simulations involving growth and interaction that are beyond the reach of QMD. In this case, however, significantly more developmental work may be needed, since applications to date have been limited and bulk assumptions and approximations often require modification near surfaces. Yet another promising application area for QBIPs is that of $f$-electron metals. The BOP and GPT/MGPT transition-metal methodologies are readily adaptable from $d$ to $f$ electrons, at least in the weak correlation DFT regime. Initial MGPT applications in this area look promising, but an added challenge is the 
structural complexity of some $f$-electron phases. Treating strongly correlated systems poses another new challenge that will require going beyond DFT to correlated-electron theories such dynamical mean field theory (DMFT) and re-building the bridge to QBIPs from that starting point. This looks possible but no work has yet been done in this area.

(iii) temperature-dependent QBIPs and direct linkage with QMD

One interesting possible use of QMD simulations is to interface them with $\mathrm{MD} / \mathrm{QBIP}$ simulations both to extend the time scale of the QMD simulations and to develop improved QBIPs at finite temperature. This linkage has actually been tried with empirical potentials in a few cases, but mostly as an interpolation mechanism and without any regard as to whether or not the potentials had physical meaning at the conditions used. For $d$ - and $f$-electron metals, however, the concept of temperature-dependent potentials is actually a very important one. In such materials there are large electronthermal effects at temperatures as low as melt arising from the high density of electronic states at the Fermi level. These effects can have a dramatic impact on high-temperature properties including the melt curve itself. Electron-thermal effects are normally treated separately from the more familiar ion-thermal effects that are associated with QBIPs constructed at zero temperature. It should be possible, however, to capture the coupled electron plus ion thermal effects simultaneously and self-consistently by building QBIPs on the basis of the total electron free energy at finite temperature. In principle, this can be done without resort to QMD simulations, but adding the possibility of refining such temperature-dependent potentials by matching QMD and MD/QBIP simulations on the fly might substantially improve the accuracy of such potentials.

\section{Acknowledgment}

This work was performed under the auspices of the U.S. Department of Energy by the University of California Lawrence Livermore National Laboratory under contract number W-7405-ENG-48. 


\section{Bibliography}

Car, R., Parrinello, M., 1985. Unified approach for molecular dynamics and densityfunctional theory. Phys. Rev. Lett. 55, 2471-74.

Dagens, L., Rasolt, M., Taylor, R., 1975. Charge densities and interionic potentials in simple metals: Nonlinear effects. II. Phys. Rev. B 11, 2726-34.

Daw, M. S., Foiles, S. M., Baskes, M. I., 1993. The embedded atom method: A review of theory and applications. Mat. Sci. Rep. 9, 251.

Finnis, M. W., Sinclair, J. E., 1984. A simple N-body potential for transition metals. Philos. Mag. A 50, 45-55.

Hafner, J., 1987. From Hamiltonians to Phase Diagrams, Springer-Verlag, Berlin.

Harrison, W. A., 1966. Pseudopotentials in the Theory of Metals, Benjamin, Reading.

Hohenberg, P, Kohn, W., 1964. Inhomogeneous electron gas. Phys. Rev. 136, B864-71.

Jacobsen, K. W., Norskov, J. K., Puska, M. J., 1987. Interatomic interactions in the effective-medium theory. Phys. Rev. B 35, 7423-42.

Kohn, W., Sham, L. J., 1965. Self-consistent equations including exchange and correlation effects. Phys. Rev. 140, A1133-38.

McMahan, A. K. and Moriarty, J. A., 1983. Structural phase stability in third-period simple metals. Phys. Rev. B 27, 3235-51.

Moriarty, J. A., 1988. Density-functional formulation of the generalized pseudopotential theory. III. Transition-metal interatomic potentials. Phys. Rev. B 38, 3199-230.

Moriarty, J. A., Phillips, R., 1991. First-principles interatomic potentials for transitionmetal surfaces. Phys. Rev. Lett. 66, 3036-39. 
Moriarty J. A., Widom, M., 1997. First-principles interatomic potentials for transitionmetal aluminides: Theory and trends across the $3 d$ series. Phys. Rev. B 56, 7905-17.

Moriarty, J. A., Vitek, V., Bulatov, V. V., Yip, S., 2002a. Atomistic simulation of dislocations and defects. J. of Computer-Aided Mater. Design 9, 99-132.

Moriarty, J. A., Belak, J. F., Rudd, R. E., Söderlind, P., Streitz, F. H., Yang, L. H., 2002 b. Quantum-based atomistic simulation of materials properties in transition metals. $J$. Phys.: Condens. Matter 14, 2825-57.

Mrovec, M., Nguyen-Manh, D., Pettifor D. G., Vitek, V., 2004. Bond-order potential for molybdenum: Application to dislocation behavior. Phys. Rev. B 69, 94115-30.

Nieminen, R. M., Puska, M. J., Manninen, M. J. (eds), 1990. Many-Atom Interactions in Solids, Springer-Verlag, Berlin.

Payne, M. C., Teter, M. P., Allan, D. C., Arias, T. A., Joannopoulos, J. D., 1992. Iterative minimization techniques for $a b$ initio total-energy calculations: molecular dynamics and conjugate gradients. Rev. Mod. Phys. 64, 1045-97

Pettifor, D. G., 1995. Bonding and Structure of Molecules and Solids, Oxford University Press, Oxford.

John A. Moriarty Lawrence Livermore National Laboratory University of California Livermore, CA 94551-0808 